\title{
Cell-based neurotrophin treatment supports long-term auditory neuron survival in the deaf guinea pig
}

\author{
Lisa N. Gillespie ${ }^{1,2}$, Mark P. Zanin ${ }^{1}$ and Robert K. Shepherd ${ }^{1,2}$ \\ ${ }^{1}$ Bionics Institute, Australia \\ ${ }^{2}$ Department of Medical Bionics, The University of Melbourne, Australia \\ Correspondence should be addressed to: \\ Dr Lisa N. Gillespie \\ Bionics Institute \\ 384 Albert Street, \\ East Melbourne, VIC 3002 \\ Australia \\ Email: Igillespie@bionicsinstitute.org \\ Phone: +61 39288 2974; Fax: +61 392882998
}

Running Title: Cell-Based Therapy for Auditory Neuron Survival

\begin{abstract}
The cochlear implant provides auditory cues to profoundly deaf patients by electrically stimulating the primary auditory neurons (ANs) of the cochlea. However, ANs degenerate in deafness; the preservation of a robust AN target population, in combination with advances in cochlear implant technology, may provide improved hearing outcomes for cochlear implant patients. The exogenous delivery of neurotrophins such as brain-derived neurotrophic factor (BDNF) and neurotrophin-3 is well known to support AN survival in deafness, and cell-based therapies provide a potential clinically viable option for delivering neurotrophins into the deaf cochlea. This study utilised cells that were genetically modified to express BDNF and encapsulated in alginate microspheres, and investigated AN survival in the deaf guinea pig following (a) cell-based neurotrophin treatment in conjunction with chronic electrical stimulation from a cochlear implant, and (b) long-term cell-based neurotrophin delivery. In comparison to deafened controls, there was significantly greater AN survival following the cell-based neurotrophin treatment, and there were ongoing survival effects for at least six months. In addition, functional benefits were observed following cell-based neurotrophin treatment and chronic electrical stimulation, with a statistically significant decrease in electrically evoked auditory brainstem response thresholds observed during the experimental period. This study demonstrates that cellbased therapies, in conjunction with a cochlear implant, shows potential as a clinically transferable means of providing neurotrophin treatment to support AN survival in deafness. This technology also has the potential to deliver other therapeutic agents, and to be used in conjunction with other biomedical devices for the treatment of a variety of neurodegenerative conditions.
\end{abstract}

Keywords: deafness; BDNF; cell therapy; cell encapsulation; cochlear implant 


\section{Introduction}

The World Health Organisation has estimated that over $5 \%$ of the world's population has a disabling hearing loss [1]. Hearing loss can range from mild to profound, and can impact upon quality of life by affecting the development of language in children, and by having adverse educational and social ramifications. People with a severe hearing loss also typically have a much higher unemployment rate and a higher rate of mental health concerns such as depression [2].

Sensorineural hearing loss (SNHL) is the most common form of deafness and effects the sensory and neural elements of the inner ear, or cochlea (Figure 1).

Specifically, SNHL is characterised firstly by a loss of auditory hair cells, which are located in the cochlea and are the first link in the chain for sound transduction, and subsequently by degeneration of the auditory neurons (ANs) which transmit the sound signals to the central auditory centres in the brain.
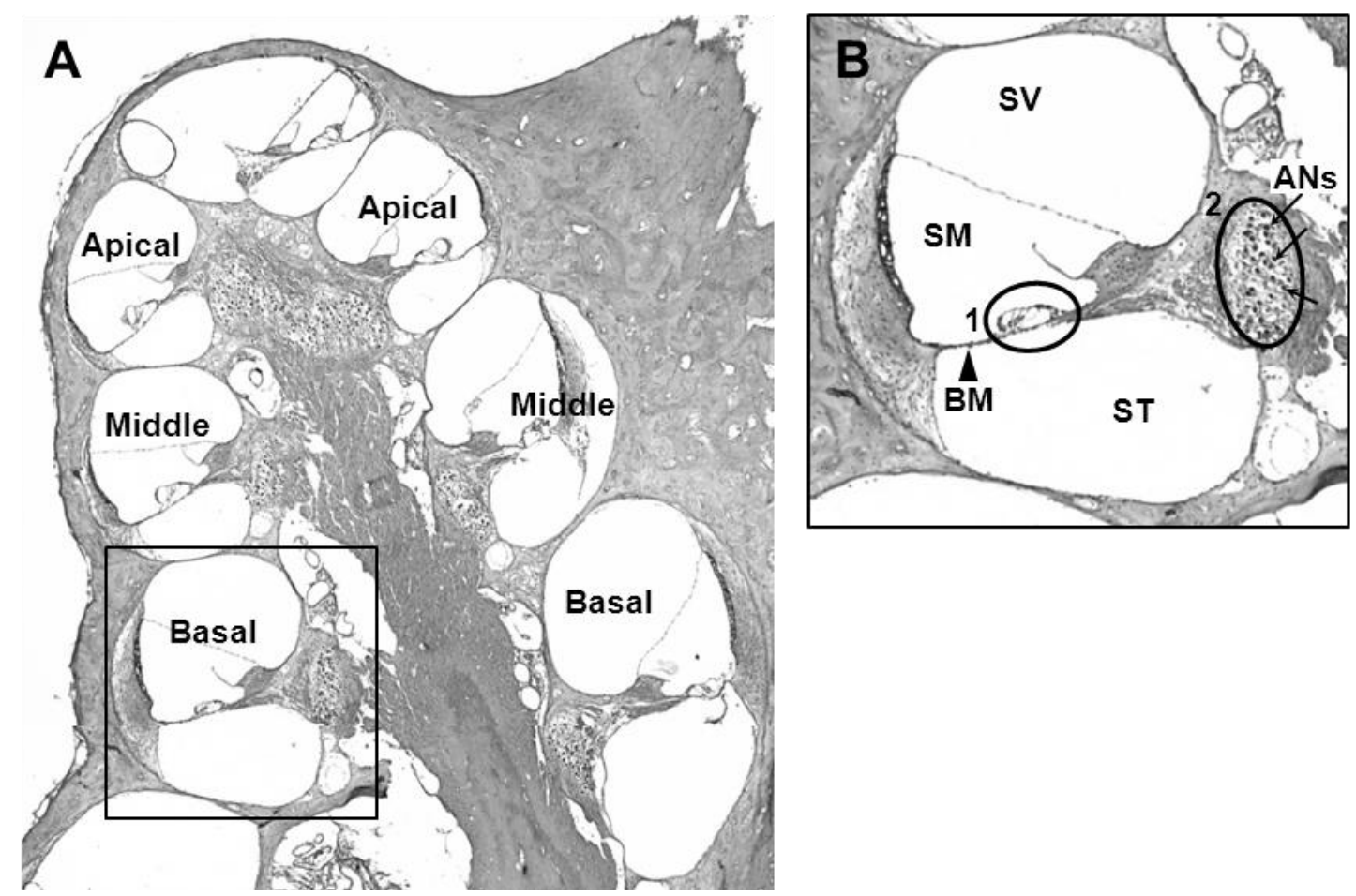

Figure 1. Anatomy of the guinea pig cochlea. (A) The inner ear, or cochlea, is a spiral structure comprised of three turns - the basal, middle and apical regions of the cochlea. (B) Depicts the region outlined in $(A)$, and shows the three chambers, or scalae, that longitudinally divide the cochlea and spiral together along its length - scala tympani (ST), scala media (SM) and scala vestibuli (SV). The auditory hair cells are the sensory cells of the cochlea and are located in the organ of Corti (1) on the basilar membrane (BM; arrowhead). The auditory neurons (ANs; arrows) are located within Rosenthal's canal (2), which spirals around the central core of the cochlea. ANs form synaptic connections with hair cells via their peripheral processes, and neural impulses generated by the hair cells in response to acoustic stimuli are transmitted by the ANs to the central auditory pathway and the auditory cortex where they are decoded, leading to the perception of sound.

Damage to or loss of hair cells can occur through a number of causes, including noise trauma, aging and ototoxic drugs. Patients with a partial SNHL can benefit from hearing aids, which amplify sound signals for acoustic stimulation of residual surviving hair 
cells. In comparison, the only therapeutic intervention for patients with a severe-toprofound SNHL is the cochlear implant, which bypasses the missing hair cells to directly electrically stimulate the ANs to provide auditory cues. However, the progressive degeneration of ANs in SNHL ultimately results in significant neuronal loss after long periods of deafness [3,4], and experimental studies indicate that this ongoing AN degeneration compromises the efficacy of cochlear implants [3,5]. Significantly, results from a recent study indicate that, for individual patients, a larger number of surviving ANs results in better performance after cochlear implantation [6], highlighting the importance of the preservation of a maximal viable population of ANs in order to optimise the clinical benefits to cochlear implant patients. In addition, future advances in cochlear implant technology, such as new stimulation strategies and electrode designs that increase the spatial resolution of stimulation, may benefit from enhanced and more viable populations of ANs [7] to provide even greater outcomes.

Neurotrophic factors, which are naturally occurring proteins important for development and maintenance of the auditory system, have been shown both in vitro and in vivo to have potential as clinically useful compounds to address SNHL. Indeed, the delivery of neurotrophins such as brain-derived neurotrophic factor (BDNF) and neurotrophin-3 (NT3) to the deaf cochlea is well known to support AN survival and prevent deafness-induced degenerative changes [8-17]. However, issues relating to safety [18] and the duration of the treatment $[19,20]$ must be addressed before the clinical translation of this therapy can be realised. Specifically, a neurotrophin delivery method designed for use in a clinical setting must be safe and biocompatible; deliver neurotrophins for extended periods in order to support long-term AN survival and function; and avoid the risks of infection associated with implantation into the cochlea [18-20].

Cell-based therapies, which would involve the long-term implantation of cells secreting a desired therapeutic in a consistent and physiologically relevant manner, have the potential to fulfil these criteria. Significantly, cell-based neurotrophin delivery has been reported to enhance AN survival in animal models of deafness [21-23]. Cell-based therapies may utilise cells that naturally secrete therapeutic agents, such as Schwann cells and choroid plexus cells, or cells that have been genetically modified to over-express specific therapeutic agents. A number of cell types, including Schwann cells and fibroblasts, have been genetically modified to express neurotrophins (for review see [24]), and long-term expression of over 12 months is possible [25]. This technique would enhance the option for autologous transplantation, whereby a recipient's own tissue could be harvested, manipulated to express the required therapeutic(s) and re-implanted, thus preventing or minimizing the risk of immunorejection.

The use of cell encapsulation technologies greatly increases the safety and biocompatibility of these therapies for use in the cochlea [21,23]. Cell encapsulation is important in order to prevent dispersal of the cells away from the site of implantation [26], and also provides an immuno-isolatory barrier, thereby enabling cell implantation without the need for co-treatment with toxic immune-suppressive drugs. Encapsulated cell-based therapies are currently being assessed in clinical trials as long-term treatments for a number of diseases. For example, populations of encapsulated choroid plexus cells $\left(\right.$ NTCELL $\left.^{\circledR}\right)$ derived from virus-free porcine colonies are currently undergoing a Phase I/Ila clinical trial for Parkinson's Disease, while encapsulated islet cells from the same source $\left(\right.$ DIABECELL ${ }^{\circledR}$ ) have progressed through Phase lla dose finding and safety and efficacy studies and are now in late-stage clinical trials for diabetes (Living Cell Technologies, Ltd; http://www.lctglobal.com; http://clinicaltrials.gov/show/NCT01734733;

http://clinicaltrials.gov/show/NCT01736228). 
Promising experimental results have also been obtained using encapsulated cellbased therapies to support AN survival in the cochlea. We have reported that Schwann cells, genetically modified to express BDNF and encapsulated in a non-biodegradable and biocompatible alginate membrane, can support AN survival in the deaf guinea pig for four weeks [21]. Furthermore, transplantation of NTCELL ${ }^{\circledR}$ into the cochlea can support AN survival in deaf cats for at least eight months [23].

The current study investigated the long-term potential of a cell-based therapy using genetically modified cells to support AN survival in deafness in conjunction with electrical stimulation from a cochlear implant. Specifically, we combined cell- and gene therapies with alginate encapsulation technology to produce encapsulated BDNF-expressing fibroblasts, and assessed the survival-promoting effects on ANs in the deaf guinea pig following implantation for periods of up to six months, and with and without chronic electrical stimulation.

\section{Experimental Procedures}

\section{Preparation of encapsulated neurotrophin-expressing cells}

Isolation and Nucleofection of fibroblasts

Fibroblasts that were nucleofected to express BDNF were used for this study based upon findings from in vitro experiments [27], which demonstrated that these parameters were most efficacious in terms of gene transfer, and produced cells which had the greatest duration of neurotrophin expression. Specifically, fibroblasts were isolated from rat sciatic nerve explants using a method similar to that used to isolate Schwann cells $[21,28]$. Briefly, 8-10 week old rats were killed using sodium pentobarbitone $(150 \mathrm{mg} / \mathrm{kg}$ intraperitoneally) and the sciatic nerves removed. The perineurium was removed and 1 $\mathrm{mm}$ segments of nerve placed into uncoated $60 \mathrm{~mm}$ tissue culture dishes with D10 culture medium, comprised of Dulbecco's Modified Eagle's Medium (DMEM; Invitrogen) containing 10\% fetal calf serum (FCS; Invitrogen), 2 mM L-glutamine (Invitrogen), 100 units $/ \mathrm{ml}$ penicillin and $100 \mathrm{mg} / \mathrm{ml}$ streptomycin (Invitrogen), and were cultured at $37^{\circ} \mathrm{C}, 5 \%$ $\mathrm{CO}_{2}$. The explants were transferred to new dishes on a weekly basis and adherent fibroblasts collected by trypsinisation. After three weeks the fibroblast cultures were pooled and purity confirmed using Thy1.1 immunocytochemistry [27].

Fibroblasts were transfected to express EGFP-tagged BDNF [29,30] using a Nucleofector II with the Basic Nucleofector Kit for Primary Mammalian Fibroblasts (Lonza). Cells at approximately $80 \%$ confluency were rinsed with PBS, trypsinised and pelleted and $1 \times 10^{6}$ cells were then used per reaction, using program T-16 as per manufacturer's instructions. Successful transfection was confirmed under direct fluorescence microscopy and selection of transformants was commenced with the addition of geneticin (G418 sulphate, $400 \mu \mathrm{g} / \mathrm{ml}$; Invitrogen) in D10. Following two weeks of selective pressure, cells were maintained in culture and subcultured weekly under $300 \mu \mathrm{g} / \mathrm{ml}$ geneticin. We have previously reported that nucleofected BDNF-expressing fibroblasts secrete BDNF for at least six months and enhance auditory neuron survival in vitro [27].

\section{Encapsulation}

Prior to encapsulation, the BDNF-fibroblasts were formed into spheres using Aggrewell ${ }^{\mathrm{TM}} 400$ plates (Stem Cell Technologies), according to manufacturers' instructions. Briefly, cells were trypsinised and resuspended in D10 culture medium 
supplemented with $10 \mu \mathrm{M}$ ROCK inhibitor (Y-27632, Sigma) to increase cell survival and promote aggregation. The Aggrewell plates are comprised of eight macrowells each containing 1200 microwells of $400 \mu \mathrm{m}$ diameter, and cells were seeded at $5 \times 10^{5}$ cells/macrowell, cultured for 24 hours and the cell clusters were then harvested.

The clusters of BDNF-fibroblasts were encapsulated (eBDNF-Fbs) in a nonbiodegradable, biocompatible alginate matrix $\left(\right.$ Immupel $\left.^{\mathrm{TM}}\right)$ courtesy of Living Cell Technologies Limited [31]. Briefly, the cell clusters were mixed with a $1.7 \%$ solution of alginate at a concentration of $1.72 \times 10^{6}$ cells $/ \mathrm{mL}$ alginate, and this mixture was pumped through a fine aperture nozzle into a bath of $1.2 \%$ calcium chloride. The resulting gelled beads were washed and serially coated with poly-L-ornithine $(0.1 \%)$, poly-L-ornithine $(0.05 \%)$ and alginate $(0.17 \%)$, and were then washed in saline and treated with sodium citrate to chelate calcium and thus liquefy the intra-capsular alginate. The resultant capsules were $500-600 \mu \mathrm{m}$ in diameter and contained 1-2 clusters of BDNF-fibroblasts. Only capsules containing 2 clusters of cells were used for implantation in the in vivo experiments. Empty alginate capsules, prepared using the same procedure but without cells, were used as controls. All capsules were maintained in $\mathrm{D} 10$ at $37^{\circ} \mathrm{C}, 5 \% \mathrm{CO}_{2}$ until required for implantation.

\section{In vivo procedures}

Twenty pigmented guinea pigs of both sexes (400-600 g) were used for this study; the treatments and durations of experiments are detailed in Table 1. All animal experiments were performed in accordance with the Code of Practice for the Care and Use of Animals for Scientific Purposes of the National Health and Medical Research Council of Australia. Experiments were performed under the approval of the Animal Research and Ethics Committee of the Royal Victorian Eye and Ear Hospital (Project Number 07/143A), Melbourne, Australia.

Table 1. Experimental Cohorts

\begin{tabular}{|ccccc|}
\hline Cohort & \multicolumn{2}{c}{ Treatment } & $\begin{array}{c}\text { Experimental } \\
\text { period }\end{array}$ & $\boldsymbol{n}$ \\
\hline $\begin{array}{c}\text { eBDNF-Fbs } \\
(1 \text { month })\end{array}$ & eBDNF-Fbs & $\begin{array}{c}\text { Empty } \\
\text { capsules }\end{array}$ & 1 month & 6 \\
\hline $\begin{array}{c}\text { eBDNF-Fbs } \\
\text { plus ES }\end{array}$ & $\begin{array}{c}\text { eBDNF-Fbs }+ \\
\text { electrode array }\end{array}$ & Untreated & 1 month & $6^{\mathrm{a}}$ \\
\hline $\begin{array}{c}\text { eBDNF-Fbs } \\
(6 \text { months })\end{array}$ & eBDNF-Fbs & $\begin{array}{c}\text { Empty } \\
\text { capsules }\end{array}$ & 6 months & 5 \\
\hline Normal hearing & none & none & N/A & $6^{\mathrm{b}}$ \\
\hline
\end{tabular}

${ }^{a}$ In one of these animals the electrode broke and so no electrical stimulation (ES) was provided and no EABRs were recorded; survival and threshold data was not quantified for this animal, however, inflammatory response was.

${ }^{\mathrm{b}} \mathrm{n}=6$ for the normal hearing cohort was derived from left and right cochleae of three animals.

\section{Ototoxin-induced deafening}

Animals were anaesthetised with a combination of intramuscular (i.m.) ketamine (40 $\mathrm{mg} / \mathrm{kg}$; Parnell Laboratories, Alexandria, NSW, Australia) and xylazine (4 mg/kg; Troy 
Laboratories, Smithfield, NSW, Australia). All animals had otoscopically normal tympanic membranes and auditory brainstem responses (ABRs) were used to confirm normal hearing. ABRs provide information about the inner ear (cochlea) and brain pathways for hearing in response to acoustic stimuli, and rely on the presence of the sensory hair cells. Normal hearing was confirmed by click-evoked auditory brainstem responses (ABRs) with thresholds of $<43$ decibels peak equivalent sound pressure level (dB p.e. SPL) [20]. One week later, animals were anaesthetised using 3-4\% isofluorane gas (APS Specialty Chemicals, Seven Hills, NSW, Australia) delivered in 100\% oxygen at $1 \mathrm{~L} / \mathrm{kg} / \mathrm{min}$ for induction and $1-1.5 \%$ isofluorane in $1 \mathrm{~L} / \mathrm{kg} / \mathrm{min}$ oxygen for maintenance. Guinea pigs were deafened via an intravenous infusion of the loop diuretic frusemide $(130 \mathrm{mg} / \mathrm{kg}$; Troy Laboratories) followed by a subcutaneous (s.c.) injection of the ototoxic aminoglycoside kanamycin sulphate (420 mg/kg; Sigma-Aldrich) [21].

\section{Implantation surgery}

Five days post-deafening, the animals were anaesthetised with ketamine (60 $\mathrm{mg} / \mathrm{kg}$, i.m.) and xylazine ( $4 \mathrm{mg} / \mathrm{kg}$, i.m.) and ABRs were performed to confirm ototoxininduced deafness. All animals exhibited a severe-profound SNHL, as indicated by clickevoked ABR thresholds of $>93 \mathrm{~dB}$ p.e. SPL in both ears. Animals then underwent implant surgery for the bilateral implantation of encapsulated BDNF-fibroblasts and empty capsules, or unilateral implantation of encapsulated BDNF-fibroblasts and a stimulating electrode array, using previously described procedures [21,23,32]. Briefly, the cochlea was exposed using aseptic surgical techniques and a small incision was made in the round window membrane. In the cells-only cohorts, the left cochleae were implanted with ten alginate capsules containing BDNF-fibroblasts, the incision in the round window membrane was sealed with fascia and the wound closed in two layers. The procedure was then repeated on the right cochleae for the implantation of ten empty capsules. These animals survived for one or six months post-implantation. For the cells plus electrical stimulation cohort, the same procedure was used to implant the animals unilaterally (left cochlea) with ten alginate capsules containing BDNF-fibroblasts plus a stimulating cochlear implant electrode array. The electrode array consisted of three platinum band electrodes on a $0.6 \mathrm{~mm}$ diameter silicone carrier. Each electrode was connected to a stainless steel leadwire system via a $25 \mu \mathrm{m}$ platinum-iridium wire (90/10) [33]. The leadwire proximal to the electrode array was fixed to the bulla using dental cement (Durelon) and to the skull using Dacron ties. The distal leadwire exited the skin via a small incision at the back of the neck. The right cochleae of each of these animals served as deafened, untreated controls. These animals survived for one month post-implantation.

\section{Chronic electrical stimulation and electrically evoked $A B R s$}

One week following implant surgery, animals in the cells plus electrical stimulation (eBDNF-Fbs plus ES) group commenced a chronic electrical stimulation program using programmable current source stimulators, as previously described [32]. Animals received stimulation 24 hours per day, seven days per week, for a total of three weeks (ie. the remainder of the one month experimental period). Electrically evoked ABRs (EABRs) were used to determine the functionality of the auditory pathway beyond the hair cells, in the deaf cochlea. In this study, EABR thresholds were recorded using the cochlear implant electrode array immediately prior to the commencement of the electrical stimulation program (day 7), and at the completion of the experimental period (day 28). EABRs were not recorded from cohorts that were not implanted with an electrode array. 


\section{Histology}

Following the experimental period, the animals were euthanized with an intraperitoneal injection of pentobarbitone sodium (160 mg/kg; Troy Laboratories) and intracardially perfused with heparinised normal saline followed by $10 \%$ neutral buffered formalin. The cochleae were harvested and decalcified in 10\% ethylenediamine-triacetic acid in $0.1 \mathrm{M}$ phosphate buffer, embedded in OCT Compound (Tissue-Tek; ProSciTech, Thuringowa, QId, Australia) and sectioned on a cryostat at $12 \mu \mathrm{m}$. Sections were stained with haematoxylin and eosin.

\section{Analysis and Statistics}

AN survival was quantified in four representative, non-consecutive mid-modiolar sections for each cochlea using a Zeiss microscope and Axiovision software. The absolute number of surviving ANs with a clear nucleus and nucleolus were counted and the crosssectional area of Rosenthal's canal from lower basal to the cochlear apex was measured, as previously described [19,21]. Data are represented as AN density (neurons $/ \mathrm{mm}^{2}$ ) for each of the basal, middle and apical cochlear regions, and are expressed as mean \pm standard error of the mean (SEM). Data are also expressed as a percentage of normal in the corresponding cochlear region. The effect of treatment on AN survival was analysed using t-tests to compare the treated cochleae versus the time-matched control cochleae for each cochlear region (basal, middle, apical).

The extent of the inflammatory response following implantation of the encapsulated cells with and without the electrode array was determined to assess the biocompatibility of the therapy. All cells, tissue and/or bone growth within the normally fluid-filled spaces of the cochlea were considered to be an inflammatory response. Using ImageJ software and previously described techniques [23], the percentage of the area of the scalae (scala tympani, scala media and scala vestibuli) occupied by inflammatory tissue was quantified for each turn (basal, middle and apical) of each cochlea. Specifically, images were converted to 8-bit greyscale and the threshold adjusted using the ImageJ 'mean' method, which segmented the images into features of interest (which appeared black) and background (white). That is, any inflammatory response or infiltrating cells appeared black, and the empty scala tympani space was white. The area of each scala was outlined, and the area occupied by the inflammatory tissue was measured and calculated as a percentage of the total scala area. A Chi-square test was used to determine the effect of treatment on the proportion of cochleae exhibiting an inflammatory response, and a oneway ANOVA was used to analyse the percentage of inflammatory response across the cochlear scalae. For all statistical analyses, data was considered statistically significant when $P<0.05$.

\section{Results}

\section{Preparation of encapsulated BDNF fibroblasts}

\section{BDNF expression}

Fluorescence microscopy confirmed that the fibroblasts were successfully nucleofected to express BDNF, with cells appearing green due to the presence of the EGFP reporter gene. In addition, successful gene transfer was confirmed by ELISA analysis of conditioned medium. Conditioned medium contained an average of $23.29 \pm$ 
$1.96 \mathrm{pg} / \mathrm{ml}$ (mean \pm SEM) BDNF per week over a course of 30 weeks, which equates to approximately $465.8 \pm 39.2 \mathrm{pg} \mathrm{BDNF} / 1 \times 10^{6}$ cells per week. The secretion profile of these cells has been characterised in detail elsewhere [27].

\section{Clustering and Encapsulation}

Cells were clustered prior to encapsulation using Aggrewell plates. After seeding into the microwells of the plates, the fibroblasts formed uniform clusters approximately 100 $\mu \mathrm{m}$ diameter and contained 400-500 cells (Figure 2A). Encapsulation produced capsules that were 500-600 $\mu \mathrm{m}$ in diameter, and contained 1-2 clusters of BDNF-fibroblasts. Each capsule used for implantation contained two clusters of cells, and therefore 800-1000 cells (Figure 2B).
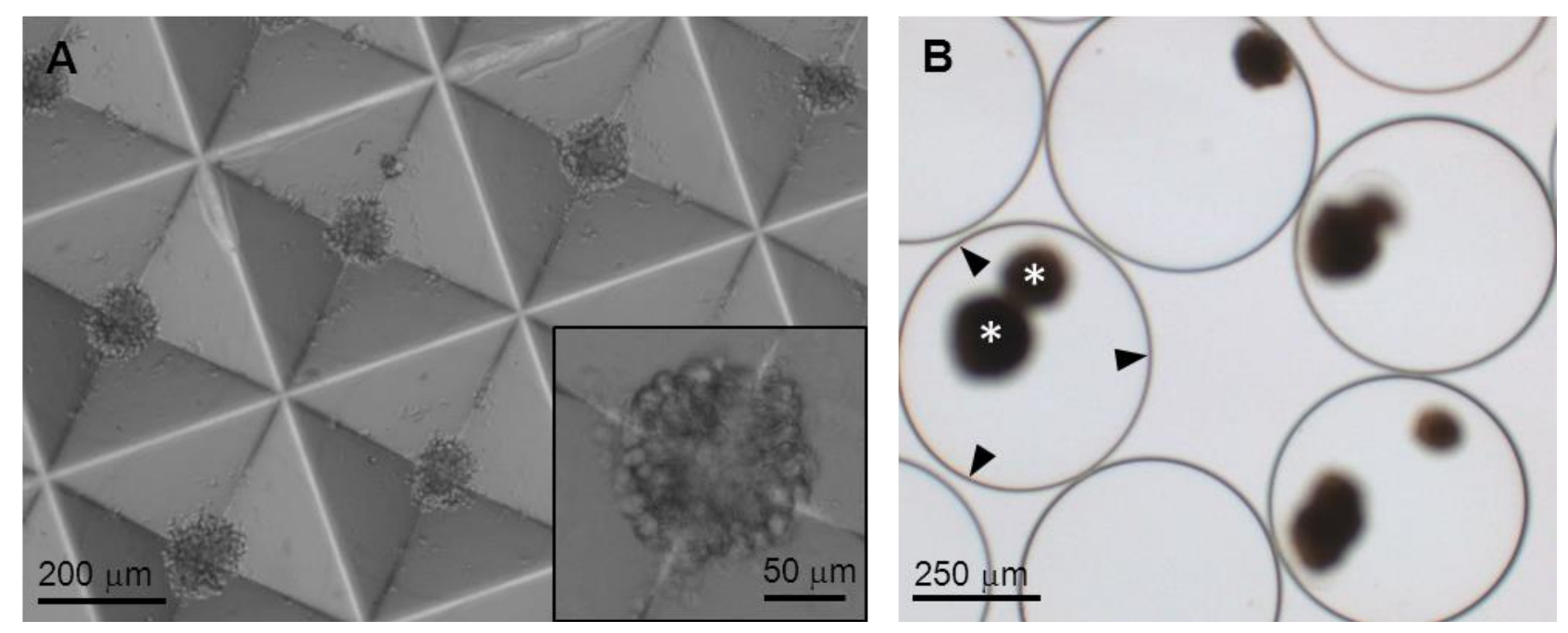

Figure 2. Clustering and encapsulation of BDNF-fibroblasts. (A) BDNF-expressing fibroblasts were clustered into spheres that were approximately $100 \mu \mathrm{m}$ in diameter and contained 400-500 cells. (B)The clustered cells $\left({ }^{*}\right)$ were subsequently encapsulated in an alginate bilayer (arrows).

\section{In vivo survival effects}

In the normal hearing cochlea, the number of ANs was similar in the basal and middle regions, with $1279 \pm 99$ and $1451 \pm 105$ (mean \pm SEM) neurons $/ \mathrm{mm}^{2}$, respectively, while slightly fewer ANs were present in the apical region $\left(1082 \pm 57\right.$ neurons $/ \mathrm{mm}^{2}$; Figure $3 ; \mathrm{NH})$.

Ototoxin exposure caused a significant loss of ANs in all cochlear regions, with $941 \pm 40$ neurons $/ \mathrm{mm}^{2}$ remaining in the basal region one month post-deafening, $957 \pm 73$ neurons $/ \mathrm{mm}^{2}$ in the middle turn, and $762 \pm 70$ neurons $/ \mathrm{mm}^{2}$ in the apical turn. This corresponds to survival rates of $73 \%, 66 \%$ and $70 \%$ in the basal, middle and apical regions, respectively, in comparison to normal hearing controls $(P<0.05$; Figure $3 ; 1 \mathrm{mo}$, UT).

Implantation of encapsulated BDNF-fibroblasts into the deaf guinea pig cochlea significantly enhanced AN survival in comparison to controls for at least six months, as shown in Figure 3. In the eBDNF-Fbs (1 month) cohort, statistically significant $(P<0.05)$ improvements in AN survival were observed in all regions of the cochlea in comparison to both deaf, untreated cochleae and deafened control cochleae implanted with empty capsules. In the basal and middle cochlear regions, cell-based neurotrophin treatment resulted in the survival of $1271 \pm 115(99 \%)$ and $1197 \pm 141$ neurons $/ \mathrm{mm}^{2}(82 \%)$, 
respectively, and there were $947 \pm 84$ surviving neurons $/ \mathrm{mm}^{2}(87 \%)$ in the apical region (Figure 3; 1 mo, Cells). In comparison, survival in the control cohort implanted with empty capsules was $963 \pm 54$ neurons $/ \mathrm{mm}^{2}(75 \%), 916 \pm 100$ neurons $/ \mathrm{mm}^{2}(63 \%)$ and $677 \pm 85$ neurons $/ \mathrm{mm}^{2}(62 \%)$ in the basal, middle and apical cochlear regions, respectively.

Importantly, one month post-implantation there was no statistical difference in the number of surviving ANs in the deaf, untreated cochleae when compared with deafened control cochleae implanted with empty capsules (Figure 3; 1mo, UT vs Empty), indicating that the surgical implantation of the capsules did not adversely influence AN survival.

Enhanced survival effects were also observed across all cochlear turns in the eBDNF-Fbs plus ES cohort in comparison to deaf, untreated cochleae and deafened controls implanted with empty capsules $(P<0.05$; Figure 3, 1mo, Cells+ES). AN survival in the basal region was $1369 \pm 96$ neurons $/ \mathrm{mm}^{2}(107 \%) ; 1252 \pm 110$ neurons $/ \mathrm{mm}^{2}(86 \%)$ in the middle turn; and $1002 \pm 86$ neurons $/ \mathrm{mm}^{2}(92 \%)$ in the apical turn. It should be noted that values greater than $100 \%$ are considered to be a result of inter-animal variation rather than regeneration of new neurons.
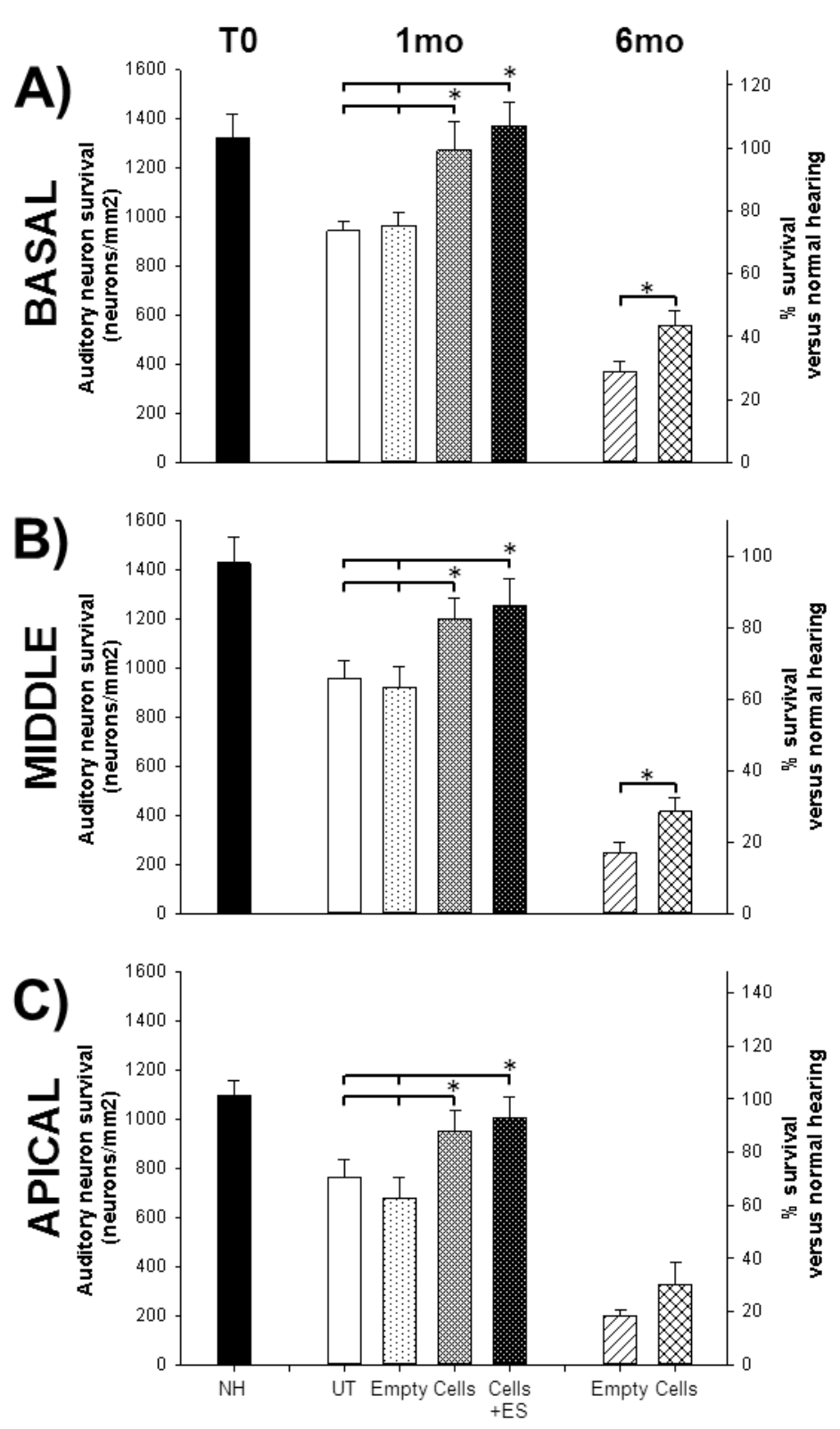

Figure 3. The effects of cell-based neurotrophin treatment on AN survival in the deaf guinea pig. Statistically significant improvements in AN survival were observed in all cochlear turns in comparison to both empty capsule and deaf, untreated controls, one month following implantation of encapsulated BDNF-fibroblasts both with and without concurrent electrical stimulation. In these cohorts, survival was maintained at nearnormal levels throughout all cochlear turns. AN survival was also significantly enhanced, in comparison to time-matched, empty capsule controls, six months postimplantation in the basal and middle cochlear turns, although overall survival rates had decreased in comparison to the one-month cohorts. Survival as a percentage of normal hearing controls is shown on the right $y$ axis. Bars = mean \pm SEM; * $P<0.05 ; \mathrm{NH}=$ normal hearing; UT=untreated; Empty=empty control capsules; Cells=encapsulated BDNFfibroblasts; $E S=$ electrical stimulation; T0=time 0 , the start of the experimental period; $1 \mathrm{mo}=1$ month post-implantation; $6 \mathrm{mo}=6$ months post-implantation. 

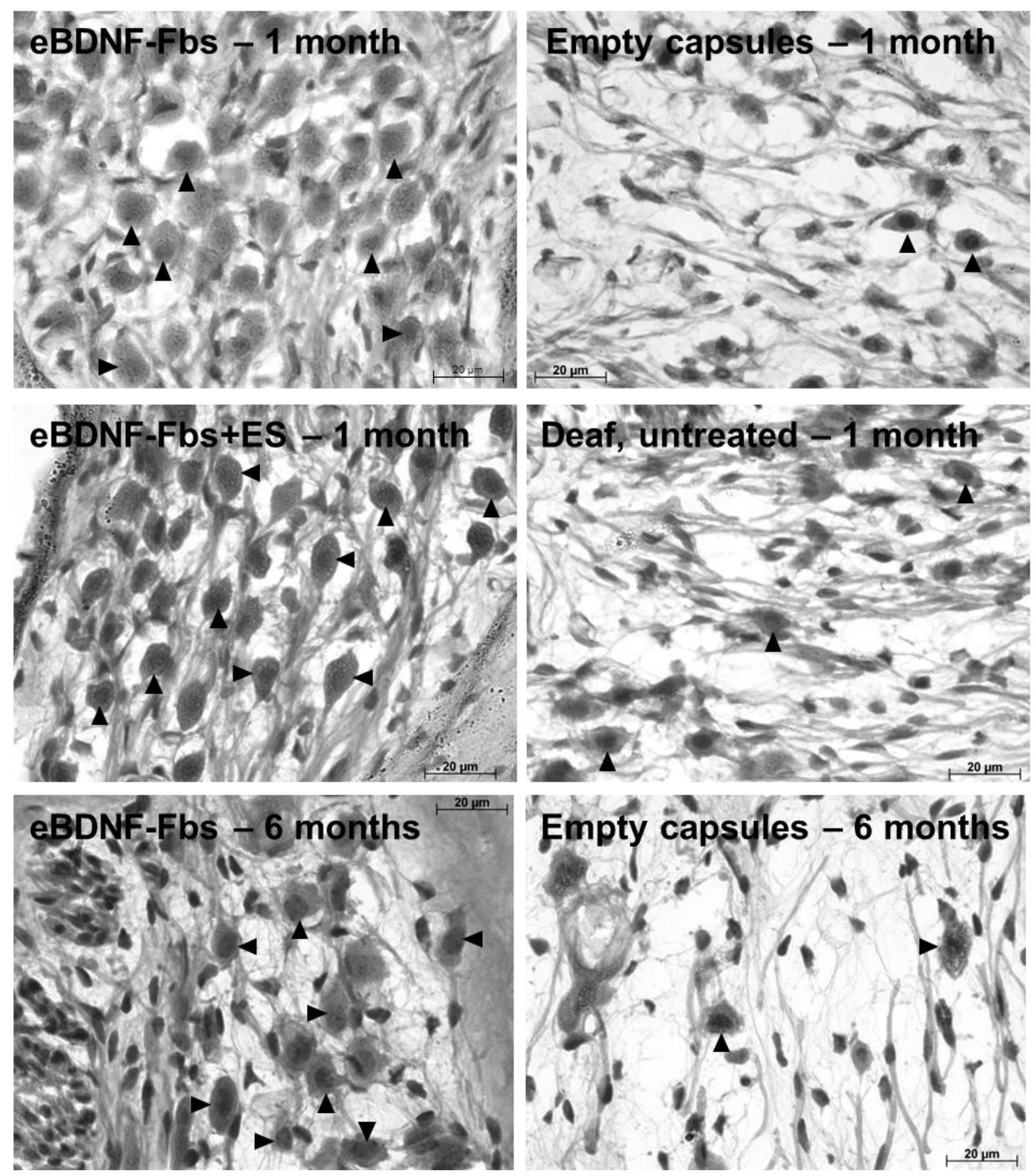

Figure 4. Photomicrographs showing AN survival in each of the experimental cohorts. Histological sections show ANs in Rosenthal's canal in the basal turn of the deaf guinea pig cochlea in each of the treatment cohorts. ANs were identified based upon the presence of an identifiable cell soma and a clear nucleus and nucleolus; examples are indicated by arrow heads. There are greater numbers of surviving ANs following the implantation of encapsulated BDNFexpressing fibroblasts (eBDNF-Fbs), alone or in combination with concurrent electrical stimulation (ES), in comparison to control, contralateral cochleae, at both one month and six months postimplantation.

In addition, in each of these cohorts with cell-based neurotrophin treatment, either with or without concurrent electrical stimulation, the AN survival rate was not statistically different to that of normal hearing controls $(P>0.05)$, indicating that in the early stages 
post-implantation the cell-based neurotrophin treatment maintained AN survival at nearnormal levels.

A significant survival effect over empty-capsule controls was also observed in the eBDNF-Fbs (6 months) cohort, with significantly greater AN survival in the basal (546 68 neurons $\left./ \mathrm{mm}^{2}\right)$ and middle $\left(414 \pm 57\right.$ neurons $\left./ \mathrm{mm}^{2}\right)$ regions than in the same regions of contralateral cochleae implanted with empty capsules for the same period of time $(P<$ 0.05 ; Figure $3 ; 6 \mathrm{mo})$. However, at this time point, survival in the apical region was not statistically different to the controls. In addition, ongoing degeneration was apparent, and when compared to normal hearing controls the survival rates in all regions of the cochlea following cell-based neurotrophin treatment ranged from $39 \%$ in the basal turn, to $25 \%$ in both the middle and apical turns. Representative images showing ANs in the basal turn of the deaf guinea pig cochlea from each of the experimental cohorts are shown in Figure 4.

\section{EABR Thresholds}

In addition to a statistically significant improvement in AN survival, the combined treatment of cell-based BDNF and chronic electrical stimulation exhibited functional benefits. Specifically, initial EABR thresholds recorded from electrode pair 1/2 on day 7 , immediately prior to the commencement of the electrical stimulation program, were $625 \pm$ $106 \mu \mathrm{A}$ (mean \pm SEM; $\mathrm{n}=5$ ). In comparison, EABR thresholds at the end of the experimental period (1 month post-deafening) were $260 \pm 37 \mu \mathrm{A}$. While we observed considerable inter-animal variability, comparison of the EABR thresholds revealed a statistically significant decrease $(P<0.05)$ in threshold from the start to the end of the experimental period, with an average threshold shift of $365 \pm 105 \mu \mathrm{A}$. EABR threshold values for recordings at the start and end of the stimulation period and subsequent threshold shifts are shown in Table 2.

Table 2. EABR thresholds

\begin{tabular}{|c|c|c|c|}
\hline GP \# & $\begin{array}{c}\text { Initial EABR } \\
\text { threshold }\end{array}$ & $\begin{array}{c}\text { Final EABR } \\
\text { threshold }\end{array}$ & Threshold shift \\
\hline GP14 & $925 \mu \mathrm{A}$ & $225 \mu \mathrm{A}$ & $-700 \mu \mathrm{A}$ \\
\hline GP15 & $350 \mu \mathrm{A}$ & $200 \mu \mathrm{A}$ & $-150 \mu \mathrm{A}$ \\
\hline GP27 & $600 \mu \mathrm{A}$ & $400 \mu \mathrm{A}$ & $-200 \mu \mathrm{A}$ \\
\hline GP28 & $450 \mu \mathrm{A}$ & $200 \mu \mathrm{A}$ & $-250 \mu \mathrm{A}$ \\
\hline GP30 & $800 \mu \mathrm{A}$ & $275 \mu \mathrm{A}$ & $-575 \mu \mathrm{A}$ \\
\hline
\end{tabular}

\section{Inflammatory reaction}

To assess the biocompatibility of the encapsulated cells following implantation into the deaf guinea pig cochlea, we quantified the total area occupied by inflammatory tissue as a percentage of the area of the scalae (tympani, media and vestibuli). Overall, we observed minimal tissue response post-implantation (Figure 5). An inflammatory response was present in 2/17 cochleae implanted with encapsulated cells, one from the cohort receiving cell-based neurotrophin treatment plus electrical stimulation and one from the eBDNF-Fbs (6 months) cohort. In addition, 1/11 cochleae implanted with empty capsules (1 month) exhibited an inflammatory response. A Chi-square analysis indicated there was 
no statistical effect of treatment on the proportion of cochleae exhibiting an inflammatory response $(P=0.681)$, and so the data was pooled for further analysis. In the eBDNF-Fbs (6 months) group, the inflammatory response was present only within the scala tympani; in the remaining two cochleae exhibiting an inflammatory response, one each from the eBDNF-Fbs plus ES and empty capsule (1 month) cohorts, the inflammatory response was more wide-spread, and was present in each scala and through all turns of the cochlea. However, the overall extent of the inflammatory response was minimal and was not statistically different across the cochlear scalae (Table 3; $P=0.825$ ). The lack of consistency between the experimental cohorts and cochlear regions in relation to the presence of an inflammatory response suggests these reactions were likely a result of individual animal sensitivities and/or specific surgical anomalies.
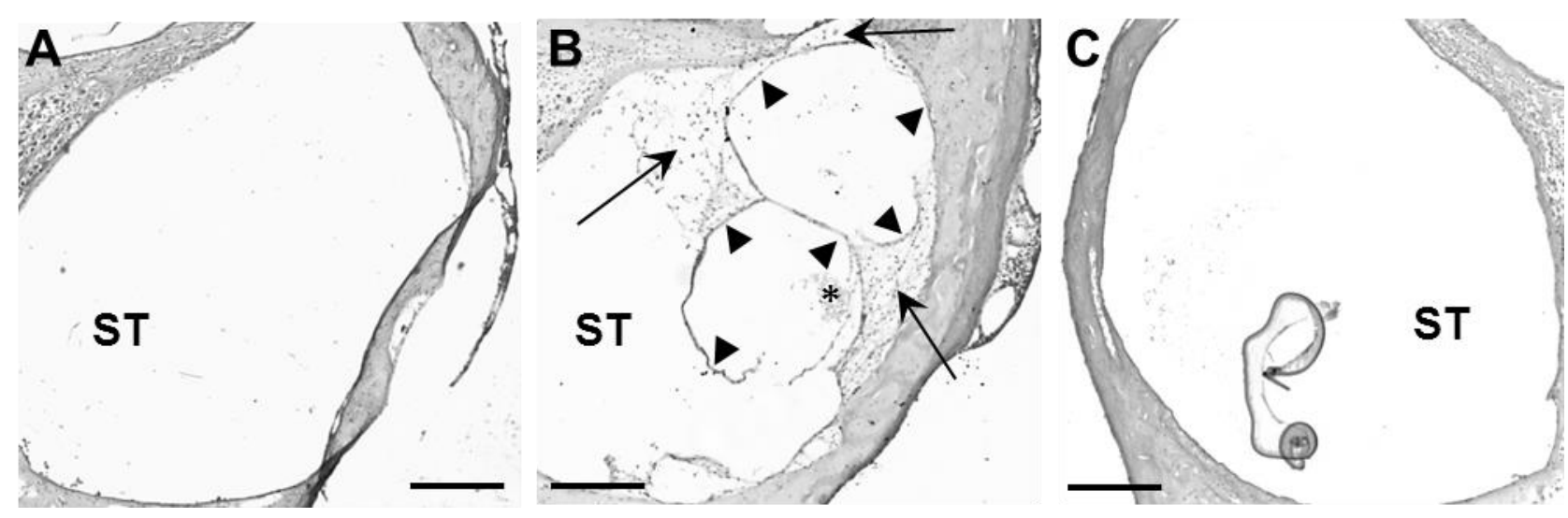

Figure 5. Minimal tissue response was observed following the implantation of encapsulated cells in the cochlea. These representative images show the varying degrees of the inflammatory/tissue response within the scala tympani (ST) that was observed following (A, B) implantation of encapsulated BDNF-fibroblasts, or (C) after implantation of empty capsules. All cells, tissue and/or bone growth within the normally fluid-filled spaces of the cochlea were considered to be an inflammatory response. The location of two capsules (arrowheads), surrounded by the inflammatory response (arrows), and a cluster of encapsulated cells $\left(^{*}\right)$ can be seen in (B). The overall tissue reaction was minimal and there was no effect of treatment condition on the proportion of cochleae exhibiting an inflammatory response $(P=0.681)$. Scale bar $=250 \mu \mathrm{m}$.

Table 3. Inflammatory response

\begin{tabular}{|cccc|}
\hline & Scala tympani & Scala media & Scala vestibuli \\
\hline $\begin{array}{c}\text { Mean \% area } \\
\text { occupied by IR }\end{array}$ & 8.36 & 5.61 & 8.86 \\
\hline SEM & 1.36 & 0.68 & 1.92 \\
\hline
\end{tabular}

$\mathrm{IR}=$ inflammatory response

\section{Discussion}

The preservation of a robust population of ANs across the frequency range of the cochlea has the potential to improve the spatial resolution of the cochlear implant, particularly associated with the use of current focussing technology [34,35], to lead to improved efficacy of the device. This study has demonstrated that the implantation of encapsulated BDNF-expressing fibroblasts into the deaf guinea pig cochlea can reduce 
the rate of AN degeneration that occurs in SNHL. While previous studies have demonstrated survival effects of neurotrophin treatment to the deaf cochlea, issues pertaining to the longevity of the survival effects and the safety of the delivery techniques used have remained. Therefore, the efficacy of a neurotrophin delivery technique for SNHL must be evaluated in terms of (i) the survival-promoting effects on ANs; (ii) the duration of the survival effects; (iii) functional outcomes; and (iv) the biocompatibility and overall safety of the treatment.

\section{Survival-promoting effects on ANs}

Cell-based neurotrophin treatment supported AN survival in the deaf guinea pig in the short-term, with significantly greater numbers of surviving ANs across all turns of the cochlea one month following implantation of encapsulated BDNF-fibroblasts, in comparison to deaf, untreated cochleae and deaf cochleae implanted with empty (control) capsules. Given that the encapsulated cells were implanted into the basal region of the cochlea, the observation of enhanced survival through all cochlear turns suggests that the BDNF secreted from the cells was able to diffuse sufficiently to elicit neuroprotective effects apically.

We have previously reported that conditioned medium from these nucleofected fibroblasts contained an average of $23.29 \pm 1.96 \mathrm{pg} / \mathrm{ml}$ (mean \pm SEM) BDNF per week over a course of 30 weeks [27], which equates to approximately $465.8 \pm 39.2 \mathrm{pg} / 1 \times 10^{6}$ cells per week. While this concentration is significantly less than the amount of BDNF secreted by Schwann cells in our previous study [21], positive effects on AN survival indicate that this is a therapeutic level. In addition, the BDNF secretion profile in the present study was much more consistent over time, which may be more conducive to sustaining AN survival over a longer time course.

The combined use of cell-based neurotrophin treatment and chronic electrical stimulation from a cochlear implant electrode array also elicited significant survival effects in comparison to empty (control) capsules, and similarly to the neurotrophin-only treatment, the survival effects were observed across all cochlear turns. However, we didn't observe survival effects following the combined treatment that were significantly greater than that of the neurotrophin-only treatment, as has been previously reported in studies where the neurotrophin was delivered via a mini-osmotic pump [20]. We consider that this is a result of differences in the concentration of BDNF delivered in the respective studies, since the amount of BDNF delivered by the cells in this study is significantly less than the amount delivered by a mini-osmotic pump.

While this study used guinea pigs and implanted them with rat fibroblasts genetically modified to express rat BDNF, the sequence and pro-form of BDNF is highly conserved across mammalian species [36]. As such, we consider that the survival effects observed in this study are analogous to the outcomes that would be achieved using alternative sources of cDNA. Furthermore, while autologous therapies could be utilized clinically, the use of cell encapsulation facilitates xenotransplantation without implications for immunorejection.

\section{Duration of survival effects}

We have demonstrated that cell-based neurotrophin treatment provides long-term protection of ANs from the degenerative effects of deafness, with encapsulated BDNFfibroblasts promoting AN survival in comparison to empty capsules for at least six months. However, at this time point, the survival effects were only observed in the basal and middle regions of the cochlea, closest to the site of implantation, which is therefore indicative of a more localized effect over this longer treatment period. In addition, it is important to note 
that while AN survival six months post-implantation was enhanced in comparison to controls, ongoing degeneration was observed, with significantly fewer surviving ANs in the six-month cohort as compared to the one-month cohort. Therefore, this treatment has slowed the rate of $A N$ degeneration in the deaf guinea pig, but has not prevented it. When considered in relation to the rate of AN degeneration across species, however, this 'slowing' effect would none-the-less translate to a positive effect over an extended timecourse in humans. Specifically, the ototoxic insult in these guinea pigs is severe and, if left untreated, causes loss of $50 \%$ of the AN population within the first $2-3$ months $[9,19,21]$. In comparison, insults to the hair cells in humans is likely in most cases to be somewhat less severe, and the subsequent neuronal degeneration takes place over a course of years [37]. As such, cell-based neurotrophin treatment is likely to result in greater numbers of surviving ANs over a longer time-course in humans, especially if used as a treatment option in conjunction with chronic electrical stimulation from a cochlear implant. Importantly, ongoing electrical stimulation following the cessation of neurotrophin treatment has previously been shown to maintain AN survival [20]. This therapy is therefore expected to further enhance the AN survival in human cochlear implant patients.

\section{Functional outcomes}

EABR measurements were recorded from animals in the cells plus electrical stimulation (eBDNF-Fbs plus ES) cohort, which were implanted with a cochlear implant electrode array and received chronic electrical stimulation for three weeks. While we could not perform these recordings in the cohorts that were not implanted with an electrode array, previous studies have demonstrated that elevated EABR thresholds are seen following ototoxin-induced SNHL, and that these thresholds increase with increased durations of deafness in both guinea pigs $[13,20]$ and other animal models of profound hearing loss [3]. Importantly, in the present study, the combination of cell-based neurotrophin treatment and electrical stimulation prevented the increases in EABR thresholds that are typically seen, indicating that the anatomical benefits in terms of AN survival had associated functional benefits.

\section{Biocompatibility and safety}

As indicated previously, in addition to efficacy in terms of long-term AN survival and function, an effective neurotrophin delivery technique must also be considered safe for use clinically. An important safety consideration for the use of a cell-based neurotrophin treatment in the cochlea is to ensure that the implanted cells don't elicit an adverse reaction, since the implantation of a foreign object into the body has the potential to elicit inflammatory tissue reactions, or even infection. For this reason, we utilized cell encapsulation technologies for this study. The advantage of this technique is that it provides an immunoisolatory barrier to protect the cells from the host immune system, and it also prevents migration of the cells away from the site of implantation. This is of particular importance in the cochlea, given that numerous studies have reported extensive cellular dispersal following transplantation into the scala tympani [38-40]. It is equally important to ensure that the encapsulation membrane is biocompatible and well tolerated within the cochlear space. Importantly, in this study the encapsulated cells were biocompatible and caused minimal inflammatory or immune response following long-term implantation in the cochlea, with no effect of treatment condition on the proportion of cochleae exhibiting an inflammatory response.

Furthermore, the stability and integrity of the capsule membrane is of importance, as degradation of the capsules within the cochlea would result in the release of the cells into the cochlear space, which could then result in diffusion of the cells away from the site 
of implantation [38-40]. The cell encapsulation in this study was performed by Living Cell Technologies, and produced alginate capsules which are not biodegradable. Previous clinical studies using this same encapsulation technique have reported successful cellular implantation into the intraperitoneal cavity with encapsulated cells remaining viable and functional for over nine years. Importantly, intact capsules could be explanted after this extended period of implantation, indicating the stability of the alginate capsule membrane [41].

The effect of the implantation of capsules into the intracochlear space also warrants discussion in relation to the size of the capsules and the cross-sectional area of the cochlea. The diameter of the capsules used in the present study $(500-600 \mu \mathrm{m})$ is similar in size to the diameter of the cochlear implant electrode array $(600 \mu \mathrm{m})$, and thus the capsules can be implanted into the cochlea to a similar depth as an electrode array [42]. It is therefore anticipated that the impact on the cochlea following implantation of these capsules would be no more significant than that seen following normal cochlear implant surgery. Indeed, in the present study, comparison of AN survival in the deaf, untreated cochleae and cochleae implanted with empty capsules show similar AN survival across all turns of the cochlea, indicating that the surgical procedure and implantation of capsules did not adversely effect AN survival.

In addition, the impact of the implantation of these capsules on the cochlear fluid mechanics is of little concern. While the transfer of vibrational signals through the cochlear fluids is necessary for auditory function in the normal cochlea with intact hair cells, the impact of impediment to vibration in the profoundly deaf ear, devoid of hair cells, is unlikely to be of any consequence.

\section{Conclusions}

Cell-based therapies have potential as a clinically viable option for delivering therapeutic agents to the cochlea in a safe, biocompatible and physiologically relevant manner. Here we demonstrate that cell-based neurotrophin treatment significantly enhances AN survival over an implant period of one month, and these survival effects translate to functional benefits with respect to electrical stimulation thresholds. In addition, cell-based neurotrophin treatment significantly reduces the rate of AN degeneration that is normally observed in SNHL over a course of six months. Furthermore, alginate encapsulated, neurotrophin-expressing cells are biocompatible following long-term implantation in the cochlea. Together, these findings add weight to the clinical feasibility of this treatment. The development of a safe and efficient technique to deliver therapeutic, otoprotective agents to the cochlea to prevent progressive AN degeneration is likely to have significant clinical benefits. In addition, cell-based therapies utilizing encapsulated genetically modified cells provide an avenue for the targeted treatment of numerous neurodegenerative conditions.

\section{Acknowledgements}

The authors would like to extend thanks to: Dr Andrew Wise and Dr James Fallon for assistance with surgery and electrophysiology; Alison Neil and Nicole Critch for technical assistance; Amy Morley, Prudence Neilsen and Ceara McGowan for histological assistance; and Marilyn Geaney and Dr Stephen Skinner from Living Cell Technologies for 
cell encapsulation. This work was funded by the Garnett Passe and Rodney Williams Memorial Foundation and the National Health and Medical Research Council of Australia (APP\# 526901). The Bionics Institute acknowledges the support it receives from the Victorian Government through its Operational Infrastructure Support Program.

\section{References}

[1] World Health Organisation, Deafness and hearing loss, http://www.who.int/mediacentre/factsheets/fs300/en/, Accessed: 10th October 2014.

[2] C.M. Li, X. Zhang, H.J. Hoffman, M.F. Cotch, C.L. Themann and M.R. Wilson, Hearing impairment associated with depression in US adults, National Health and Nutrition Examination Survey 2005-2010, JAMA Otolaryngol Head Neck Surg 140 (2014) 293-302.

[3] N.A. Hardie and R.K. Shepherd, Sensorineural hearing loss during development: morphological and physiological response of the cochlea and auditory brainstem, Hear Res 128 (1999) 147-165.

[4] P.A. Leake and G.T. Hradek, Cochlear pathology of long term neomycin induced deafness in cats, Hear Res 33 (1988) 11-33.

[5] R.K. Shepherd and E. Javel, Electrical stimulation of the auditory nerve. I. Correlation of physiological responses with cochlear status, Hear Res 108 (1997) 112-144.

[6] M. Seyyedi, L.M. Viana and J.B. Nadol, Jr., Within-subject comparison of word recognition and spiral ganglion cell count in bilateral cochlear implant recipients, Otol Neurotol 35 (2014) 1446-1450.

[7] A.K. Wise and L.N. Gillespie, Drug delivery to the inner ear, J Neural Eng 9 (2012) 065002.

[8] P. Ernfors, M.L. Duan, W.M. EIShamy and B. Canlon, Protection of auditory neurons from aminoglycoside toxicity by neurotrophin-3, Nat Med 2 (1996) 463-467.

[9] L.N. Gillespie, G.M. Clark and P.L. Marzella, Delayed neurotrophin treatment supports auditory neuron survival in deaf guinea pigs, Neuroreport 15 (2004) 1121-1125.

[10] S.L. McGuinness and R.K. Shepherd, Exogenous BDNF rescues rat spiral ganglion neurons in vivo, Otol Neurotol 26 (2005) 1064-1072.

[11] J.M. Miller, D.H. Chi, L.J. O'Keeffe, P. Kruszka, Y. Raphael and R.A. Altschuler, Neurotrophins can enhance spiral ganglion cell survival after inner hair cell loss, Int J Dev Neurosci 15 (1997) 631-643.

[12] R.T. Richardson, A.K. Wise, B.C. Thompson, B.O. Flynn, P.J. Atkinson, N.J. Fretwell, J.B. Fallon, G.G. Wallace, R.K. Shepherd, G.M. Clark and S.J. O'Leary, Polypyrrolecoated electrodes for the delivery of charge and neurotrophins to cochlear neurons, Biomaterials 30 (2009) 2614-2624.

[13] T. Shinohara, G. Bredberg, M. Ulfendahl, I. Pyykko, N.P. Olivius, R. Kaksonen, B. Lindstrom, R. Altschuler and J.M. Miller, Neurotrophic factor intervention restores auditory function in deafened animals, Proc Natl Acad Sci U S A 99 (2002) 1657-1660.

[14] H. Staecker, R. Kopke, B. Malgrange, P. Lefebvre and T.R. Van de Water, NT-3 and/or BDNF therapy prevents loss of auditory neurons following loss of hair cells, Neuroreport 7 (1996) 889-894. 
[15] A.K. Wise, C.R. Hume, B.O. Flynn, Y.S. Jeelall, C.L. Suhr, B.E. Sgro, S.J. O'Leary, R.K. Shepherd and R.T. Richardson, Effects of localized neurotrophin gene expression on spiral ganglion neuron resprouting in the deafened cochlea, Mol Ther 18 (2010) 11111122.

[16] A.K. Wise, R. Richardson, J. Hardman, G. Clark and S. O'Leary, Resprouting and survival of guinea pig cochlear neurons in response to the administration of the neurotrophins brain-derived neurotrophic factor and neurotrophin-3, J Comp Neurol 487 (2005) 147-165.

[17] T. Yamagata, J.M. Miller, M. Ulfendahl, N.P. Olivius, R.A. Altschuler, I. Pyykko and G. Bredberg, Delayed neurotrophic treatment preserves nerve survival and electrophysiological responsiveness in neomycin-deafened guinea pigs, J Neurosci Res 78 (2004) 75-86.

[18] R.K. Shepherd, Rescuing the cochlea: the challenges, ENT \& audiology news 19 (2011) 49-52.

[19] L.N. Gillespie, G.M. Clark, P.F. Bartlett and P.L. Marzella, BDNF-induced survival of auditory neurons in vivo: Cessation of treatment leads to an accelerated loss of survival effects, J Neurosci Res 71 (2003) 785-790.

[20] R.K. Shepherd, A. Coco, S.B. Epp and J.M. Crook, Chronic depolarisation enhances the trophic effects of BDNF in rescuing auditory neurons following a sensorineural hearing loss, J Comp Neurol 486 (2005) 145-158.

[21] L.N. Pettingill, A.K. Wise, M.S. Geaney and R.K. Shepherd, Enhanced auditory neuron survival following cell-based BDNF treatment in the deaf guinea pig, PLoS One 6 (2011) e18733.

[22] D. Rejali, V.A. Lee, K.A. Abrashkin, N. Humayun, D.L. Swiderski and Y. Raphael, Cochlear implants and ex vivo BDNF gene therapy protect spiral ganglion neurons, Hear Res 228 (2007) 180-187.

[23] A.K. Wise, J.B. Fallon, A.J. Neil, L.N. Pettingill, M.S. Geaney, S.J. Skinner and R.K. Shepherd, Combining cell-based therapies and neural prostheses to promote neural survival, Neurotherapeutics 8 (2011) 774-787.

[24] M.P. Zanin, L.N. Pettingill, A.R. Harvey, D.F. Emerich, C.G. Thanos and R.K. Shepherd, The development of encapsulated cell technologies as therapies for neurological and sensory diseases, J Control Release 160 (2012) 3-13.

[25] S.R. Winn, M.D. Lindner, A. Lee, G. Haggett, J.M. Francis and D.F. Emerich, Polymer-encapsulated genetically modified cells continue to secrete human nerve growth factor for over one year in rat ventricles: behavioral and anatomical consequences, Exp Neurol 140 (1996) 126-138.

[26] B.A. Nayagam, S.S. Backhouse, C. Cimenkaya and R.K. Shepherd, Hydrogel limits stem cell dispersal in the deaf cochlea: implications for cochlear implants, J Neural Eng 9 (2012) doi: 10.1088/1741-2560/1089/1086/065001.

[27] M.P. Zanin, M. Hellström, R.K. Shepherd, A.R. Harvey and L.N. Gillespie, Development of a cell-based treatment for long-term neurotrophin expression and spiral ganglion neuron survival, Neuroscience 277 (2014) 690-699.

[28] J.P. Brockes, K.L. Fields and M.C. Raff, Studies on cultured rat Schwann cells. I. Establishment of purified populations from cultures of peripheral nerve, Brain Res 165 (1979) 105-118. 
[29] T. Brigadski, M. Hartmann and V. Lessmann, Differential vesicular targeting and time course of synaptic secretion of the mammalian neurotrophins, J Neurosci 25 (2005) 76017614.

[30] W. Haubensak, F. Narz, R. Heumann and V. Lessmann, BDNF-GFP containing secretory granules are localized in the vicinity of synaptic junctions of cultured cortical neurons, J Cell Sci 111 ( Pt 11) (1998) 1483-1493.

[31] S.J. Skinner, M.S. Geaney, H. Lin, M. Muzina, A.K. Anal, R.B. Elliott and P.L. Tan, Encapsulated living choroid plexus cells: potential long-term treatments for central nervous system disease and trauma, J Neural Eng 6 (2009) 065001.

[32] R.K. Shepherd, A. Coco and S.B. Epp, Neurotrophins and electrical stimulation for protection and repair of spiral ganglion neurons following sensorineural hearing loss, Hear Res 242 (2008) 100-109.

[33] R. Shepherd and J. Xu, A multichannel scala tympani electrode array incorporating a drug delivery system for chronic intracochlear infusion, Hear Res 172 (2002) 92-98.

[34] C. van den Honert and D.C. Kelsall, Focused intracochlear electric stimulation with phased array channels, J Acoust Soc Am 121 (2007) 3703-3716.

[35] S.S. George, A.K. Wise, M.N. Shivdasani, R.K. Shepherd and J.B. Fallon, Evaluation of focused multipolar stimulation for cochlear implants in acutely deafened cats., Journal of Neural Engineering (in press)

[36] P.C. Maisonpierre, M.M. Le Beau, R. Espinosa, 3rd, N.Y. Ip, L. Belluscio, S.M. de la Monte, S. Squinto, M.E. Furth and G.D. Yancopoulos, Human and rat brain-derived neurotrophic factor and neurotrophin-3: gene structures, distributions, and chromosomal localizations, Genomics 10 (1991) 558-568.

[37] J.B. Nadol, Jr., Y.S. Young and R.J. Glynn, Survival of spiral ganglion cells in profound sensorineural hearing loss: implications for cochlear implantation, Ann Otol Rhinol Laryngol 98 (1989) 411-416.

[38] B. Coleman, J. Hardman, A. Coco, S. Epp, M. de Silva, J. Crook and R.K. Shepherd, Fate of embryonic stem cells transplanted into the deafened mammalian cochlea, Cell Transplantation 15 (2006) 369-380.

[39] Z. Hu, M. Ulfendahl and N.P. Olivius, Survival of neuronal tissue following xenograft implantation into the adult rat inner ear, Exp Neurol 185 (2004) 7-14.

[40] P. Olivius, L. Alexandrov, J. Miller, M. Ulfendahl, D. Bagger-Sjoback and E.N. Kozlova, Allografted fetal dorsal root ganglion neuronal survival in the guinea pig cochlea, Brain Res 979 (2003) 1-6.

[41] R. Elliott, L. Escobar, P. Tan, M. Muzina and S. Zwain, Live encapsulated porcine islets from a type 1 diabetic patient $9.5 \mathrm{yr}$ after xenotransplantation, Xenotransplantation 14 (2007) 157-161.

[42] B.J. Gantz, C. Turner, K.E. Gfeller and M.W. Lowder, Preservation of hearing in cochlear implant surgery: advantages of combined electrical and acoustical speech processing, Laryngoscope 115 (2005) 796-802. 


\section{University Library}

\section{- M M N E R VA A gateway to Melbourne's research publications}

Minerva Access is the Institutional Repository of The University of Melbourne

Author/s:

Gillespie, LN;Zanin, MP;Shepherd, RK

Title:

Cell-based neurotrophin treatment supports long-term auditory neuron survival in the deaf guinea pig

Date:

2015-01-28

Citation:

Gillespie, L. N., Zanin, M. P. \& Shepherd, R. K. (2015). Cell-based neurotrophin treatment supports long-term auditory neuron survival in the deaf guinea pig. JOURNAL OF CONTROLLED RELEASE, 198, pp.26-34. https://doi.org/10.1016/j.jconrel.2014.11.026.

Persistent Link:

http://hdl.handle.net/11343/43185 